\title{
Presentation, Management and Outcome of Acute Sigmoid Diverticulitis Requiring Hospitalization
}

\author{
Jose A. Alvarez Ricardo F. Baldonedo Isabel G. Bear Jorge Otero Gerardo Pire \\ Paloma Alvarez Jose I. Jorge \\ Service of General Surgery, Hospital San Agustín, Avilés, Spain
}

\section{Key Words}

Diverticular disease $\cdot$ Colonic diverticulitis $\cdot$ Fistula

\begin{abstract}
Aim: This study was conducted to assess the presentation, management, and outcomes of patients with acute sigmoid diverticulitis requiring hospitalization. Methods: From 1986 to 2005 , the medical records of 265 patients treated for acute sigmoid diverticulitis requiring hospitalization were retrospectively analyzed. Data were collected with regard to patient's demographics, clinical characteristics, presentations of acute diverticulitis, treatment, morbidity, and mortality. Results: Only 47 patients (17.7\%) had a previous diverticulitis episode. Of the 265 patients, 166 (62.6\%) were managed without operation, and 99 (37.4\%) underwent surgery. Overall and major morbidity in the whole series were $30.2(80 / 265)$ and $15.5 \%$ (40/265), respectively; whereas among the patients with surgical management, were 72.7 (72/99), and $35.3 \%$ (35/99), respectively. Overall and postoperative mortality rates were $2.6(7 / 265)$ and $6.1 \%(6 / 99)$, respectively. Older age, steroid use, perforation, and co-morbidities were significantly associated with unfavorable outcomes. Conclusions: It was concluded that surgery for acute sigmoid diverticulitis requiring hospitalization carries important morbid-
\end{abstract}

\section{KARGER}

Fax +41613061234

E-Mail karger@karger.ch

www.karger.com (c) 2007 S. Karger AG, Basel

0253-4886/07/0246-0471\$23.50/0

Accessible online at:

www.karger.com/dsu ity and mortality. To achieve improvements in outcome, a selective therapeutic approach should be considered, choosing the best surgical procedure for each complication of diverticular disease.

Copyright $\odot 2007$ S. Karger AG, Basel

\section{Introduction}

Colonic diverticulosis is among the most common diseases of developed Western countries. While most people with diverticular disease remain asymptomatic, between 10 and $25 \%$ of patients with diverticulosis will ultimately developed diverticulitis. Of those patients who develop diverticulitis, 15 to $20 \%$ will present significant complications, such as phlegmon, abscess, fistula, obstruction, bleeding, or perforation $[1,2]$. Complicated diverticulitis remains a significant cause of morbidity and mortality in patients afflicted with this problem. This morbidity and mortality depend, not so much on the operative procedure, but on the severity of the disease and the associated comorbid conditions, namely the presence of fecal or purulent peritonitis, past medical problems, immune status, and nutritional status [3].

Dr. José Antonio Alvarez Camino de Heros, 4

ES-33400 Avilés (Spain)

Tel. +34 985123 000, Fax +34 985123 052, E-Mail josealvar@telecable.es 
The management of patients with acute diverticulitis is largely dictated by the stage of the disease at the time of presentation and by the observed response to the initiated treatment [4]. Thus, although most patients with acute diverticulitis respond to conservative measures including bowel rest and antibiotics, some will have persistent symptoms or complications and require surgery. The operative approach for diverticular emergencies has evolved slowly, stimulated by the advent of effective antibiotics and by improvements in resuscitative and anesthetic techniques. Nowadays, it is widely accepted that surgery should be considered when complications of diverticular disease occur.

Advances in diagnostic modalities, medical therapy, and surgical techniques have changed the management and outcome of diverticulitis in recent years. The aim of this study was to update our understanding of the treatment, morbidity, mortality, characteristics, and outcomes of patients with acute diverticulitis requiring hospitalization in the current era.

\section{Patients and Methods}

\section{Study Design}

Between January 1986 and December 2005, the charts of all patients that presented with acute sigmoid diverticulitis requiring hospitalization in a single district general hospital were retrospectively reviewed. We defined acute sigmoid diverticulitis requiring hospitalization as the presence of documented acute diverticulitis associated with phlegmon (in patients too ill for outpatient antibiotic therapy alone), perforation, abscess, obstruction or fistula. For the purposes of the present study, we defined patients as suffering from acute diverticulitis if they were acutely admitted with lower abdominal pain and displayed tenderness and/or guarding in the left iliac fossa. They also had to show evidence of a systemic inflammatory response which was indicated by the presence of one or more of the following findings: temperature $>38^{\circ} \mathrm{C}$, white blood cell count $>11.0 \times 10^{9} / 1$, and C-reactive protein $>10 \mathrm{mg} / \mathrm{l}$. The diagnosis of diverticular disease was confirmed (and other pathology excluded) in all patients on subsequent investigations which included a combination of one or more of the following diagnostic methods: contrast enema, computed tomography (CT) scan, and colonoscopy. All patients had involvement of the sigmoid colon only. Other sites of involvement, and patients who underwent operation for bleeding secondary to diverticular disease were not included.

\section{Data Collection and Definitions}

Data were collected with regard to patient's demographics, significant concomitant illnesses, symptoms and physical findings, prior history of diverticulitis, type of complicated diverticulitis, surgical and medical treatment, morbidity, and mortality. Determination of type of complicated diverticultis was based on the following definitions: phlegmon was a symptomatic inflamma- tory mass not associated with pus; pericolic abscess was a collection of purulent material localized near the colon; distant abscess was defined as a pelvic, abdominal or retroperitoneal abscess; generalized peritonitis was established by the presence of free pus or feces in the peritoneal cavity at laparotomy; fistulas included colovaginal, colovesical, or colocutaneous connections, and obstruction was characterized by obstructive symptoms and a stricture diagnosed by radiologic studies or pathologic evaluation. The term perforation was applied in patients who had pericolic abscess, distant abscess, or generalized peritonitis. The choice of operation was made by the surgeon at the time of the operation based on the condition of the patient, status of the abdomen, blood supply of the bowel, and experience of the operating team. Overall morbidity was equal to the sum of nonfatal morbidity and mortality. Major complications were defined as those affecting major organ systems. Mortality was defined as death within 30 days of operation, or during the same hospitalization if longer than 30 days.

\section{Statistical Analysis}

The data were compiled and analyzed by using the program software SPSS for Windows, Version 10.0 (SPSS, Chicago, Ill., USA). Categorical variables were compared with the $\chi^{2}$ test or Fisher's exact test, where appropriate. Continuous, non-parametric distributed variables were analyzed with the Mann-Whitney U test. Normally distributed data were analyzed with Student's $t$ test. Differences were considered significant at $\mathrm{p}<0.05$.

\section{Results}

After exclusion criteria were applied, 265 patients that presented with acute sigmoid diverticulitis requiring hospitalization were identified and analyzed. Of these patients, 132 were men and 133 were women. The median age of patients was 64 (31-92) years. Forty-seven patients $(17.7 \%)$ were less than 50 years of age. The proportion of patients less than 50 years in the first 10 years and in the last 10 years of the study was the same (17.7\%). At least one significant concomitant illness was identified in 131 patients (49.4\%) (table 1). Nine patients (3.4\%) were taking steroids at the time of their diverticulitis episode for a variety of indications. Presentations of acute diverticulitis requiring hospitalization observed on admission included acute phlegmon in 136 patients (51.3\%), pericolic abscess in 37 patients (13.9\%), distant abscess in 22 patients (8.3\%), generalized peritonitis in 55 patients (20.7\%), of whom 50 were purulent and 5 faecal, fistula in 9 patients (3.4\%), and obstruction/stricture in $6(2.3 \%)$. In terms of location of fistulas, seven patients had colovesical fistulas, one patient had a colovaginal fistula, and another one had a colocutaneous fistula. Overall, 114 patients (43\%) presented with perforation. Only 47 patients $(17.7 \%)$ had a history of at least one prior diverticulitis 
episode, whereas $218(82.3 \%)$ presented with acute sigmoid diverticulitis requiring hospitalization as their initial event. The majority of patients presented with abdominal pain, 211 cases (79.6\%) and fever, 149 cases (56.2\%). A palpable mass in the left lower abdomen was found in 18 patients (6.8\%). In total, a CT scan was performed in 89 patients (33.6\%) as part of their evaluation. This diagnostic method was performed in 40 of the 136 patients with phlegmon formation, and in 31 of the 59 of the patients with abscess formation.

Of the 265 patients, $166(62.6 \%)$ were treated conservatively without operation, and 99 (37.4\%) underwent surgical treatment. Management of the various presentations of acute diverticulitis is summarized in table 2 . Acute phlegmon was generally managed without operation, but five patients underwent surgical intervention. Of these, one had suspected appendicitis. Surgery in this patient may have been avoidable. All other laparotomies were performed because of continuing symptoms of infection by acute diverticulitis. Most patients with pericolic abscess were treated conservatively, and only 8 patients underwent surgery. In these patients, failed conservative management by persistent sepsis was the indication for surgery. All but 4 patients with a distant abscess underwent a colonic resection. Primary anastomosis was performed in 8 of these patients, in 2 of whom the abscesses were percutaneously and transrectally drained before surgery. Fourteen of the 26 patients diagnosed with abscess formation (53.8\%) that went to the operating room had known abscess formation prior to their operation. These patients presented different contraindications to undergo a percutaneous drainage. Thus, in some of these patients an inflammatory cancer could not be excluded. In others, surgical aspects linked to the patient's severity or radiologic contraindications in relation to size or location of the abscesses were the reasons that explained this fact. Patients with generalized peritonitis were usually managed by Hartmann's resection, and only one seriously sick patient underwent drainage alone. $\mathrm{Pa}$ tients with obstruction were mainly managed with surgery. The principle operation for fistula was segmental resection and primary anastomosis.

The overall and major morbidity rates for the cohort were 30.2 (80/265) and 15.5\% (40/265), respectively. Among the patients with surgical management, 72 (72.7\%) had some type of complication, and 35 (35.3\%) had major complications (tables 2, 3). The anastomotic leak rate was $8.3 \%(2 / 24)$. The 2 patients who developed postoperative anastomotic leaks after resection and primary anastomosis presented on admission as a colovesi-

Table 1. Important coexistent diseases in 131 patients

\begin{tabular}{ll}
\hline Associated disease & $\mathrm{n}$ \\
\hline Arterial hypertension & $69(26.0 \%)$ \\
Cardiovascular disorders & $41(15.5 \%)$ \\
$\quad$ Previous myocardial infarction & 21 \\
$\quad$ Atrial fibrillation & 10 \\
$\quad$ Valvular heart disease & 5 \\
$\quad$ Congestive heart failure & 3 \\
Myocardiopathy & 2 \\
Chronic obstructive pulmonary disease & $19(7.2 \%)$ \\
Diabetes mellitus & $18(6.8 \%)$ \\
Collagen-vascular disease & $12(4.5 \%)$ \\
Malignancy & $7(2.6 \%)$ \\
Previous stroke & $7(2.6 \%)$ \\
Cirrhosis & $5(1.9 \%)$ \\
\hline
\end{tabular}

${ }^{\text {a }}$ Patients with recent malignancy diagnosis or metastatic disease and receiving treatment.
Table 2. Surgical treatment and postoperative major morbidity

\begin{tabular}{lcllll}
\hline Presentation & $\begin{array}{l}\text { No } \\
\text { operation }\end{array}$ & $\begin{array}{l}\text { Drainage } \\
\text { alone }\end{array}$ & $\begin{array}{l}\text { Loop } \\
\text { stoma }\end{array}$ & $\begin{array}{l}\text { Resection and } \\
\text { primary } \\
\text { anastomosis }\end{array}$ & $\begin{array}{l}\text { Hartmann's } \\
\text { procedure }\end{array}$ \\
\hline $\begin{array}{l}\text { Acute phlegmon } \\
\text { Pericolic abscess }\end{array}$ & 131 & $1(1)$ & 1 & $2(1)$ & 1 \\
Distant abscess & 29 & - & - & 4 & $4(1)$ \\
Purulent peritonitis & 4 & - & - & $8(3)$ & $10(2)$ \\
Fecal peritonitis & - & $1(1)$ & - & $1^{\mathrm{a}}$ & $48(18)$ \\
Large bowel obstruction & - & - & - & - & $5(4)$ \\
Fistula & 2 & - & 1 & $2(1)$ & 1 \\
\hline
\end{tabular}

Numbers of major complications appear in parentheses.

a The patient had proximal diverting colostomy and primary anastomosis. 
cal fistula and as a distant abscess, respectively. Fiftynine patients (59.6\%) developed wound infection and 6 wound dehiscence. Older age ( $>70$ years), steroid use, significant preexisting comorbidities, and perforated diverticulitis were all significantly associated with increased postoperative major morbidity $(\mathrm{p}=0.014, \mathrm{p}=0.003, \mathrm{p}<$ 0.001 and $\mathrm{p}<0.001$, respectively). Four patients had a postoperative complication that required re-operation. Of these, 2 underwent drainage of intra-abdominal abscesses after Hartmann's procedures. Another patient necessitated re-operation for adhesive small bowel obstruction subsequent to resection and primary anastomosis. In the remaining patient, an anastomotic leakage also after a primary anastomosis was treated by colostomy. The deaths in the whole series including patients treated conservatively and those who underwent surgery were 7 , which is $2.6 \%$ of all patients. Six of the deaths followed surgery (6.1\%). The remaining patient who died had been managed conservatively without surgery. Mortality was associated with perforation at presentation in $85.7 \%$ of cases (6/7). The patient who died before surgical intervention was aged, had important coexistent co-morbidity and died from uncontrolled sepsis as consequence of a pericolic abscess. The stated cause of death in the 6 surgical patients was persistent abdominal sepsis (3), cardiac failure (1), pulmonary embolism (1), and bronchopneumonia (1). Septic complications accounted for $66.7 \%$ of the observed deaths (4/6). Steroid use was significantly associated with the perforation rate $(\mathrm{p}=0.03)$, but not with the mortality rate $(\mathrm{p}=0.238)$. Older age ( $>70$ years $)$ was significantly associated with increased mortality $(p=0.005)$. One of 2 patients who had anastomotic leak died, but the cause of death was a nondisease-related complication. Coexistent disease such as diabetes, cardiovascular diseases, and chronic obstructive pulmonary disease was significantly associated with death $(\mathrm{p}=0.033$, $\mathrm{p}=0.007$ and $\mathrm{p}=0.017$, respectively).

\section{Discussion}

Although diverticular disease is a very common and frequently encountered condition, a lack of clarity in the definition of acute diverticulitis has led to difficulty in interpreting study results and comparing outcomes for complicated diverticular disease [5]. Patients can present with the disease at any point on the spectrum of care. In the past, patients presented later in the care process and surgery was reserved primarily for those critically sick because of a high mortality [1]. Nowadays, several factors
Table 3. Major complications after surgery for acute diverticulitis requiring hospitalization

\begin{tabular}{lc}
\hline Complication & $\mathrm{n}$ \\
\hline Gastrointestinal & $24(24.2 \%)$ \\
Prolonged ileus & 6 \\
Intra-abdominal abscess & 6 \\
Persistent peritonitis & 5 \\
Ischemia/breakdown of colostomy & 3 \\
Anastomotic leak & 2 \\
Upper digestive hemorrhage & 1 \\
Adhesive intestinal obstruction & 1 \\
Respiratory & $11(11.1 \%)$ \\
Pneumonia & 5 \\
Pneumothorax & 3 \\
Adult respiratory distress syndrome & 1 \\
Chronic obstructive pulmonary disease & 1 \\
Pulmonary embolism & 1 \\
Renal & $10(10.1 \%)$ \\
Acute renal failure & 10 \\
Cardiac & $6(6.1 \%)$ \\
Congestive heart failure & 3 \\
Paroxystic atrial fibrillation & 3 \\
Other & $2(2.0 \%)$ \\
Catheter-related sepsis & 1 \\
Cerebrovascular accident & 1 \\
\hline
\end{tabular}

More than one complication per patient is possible.

such as increasing public awareness, better access to medical information, and improved surgical techniques, have made possible that patients are apt to present earlier to the medical system. In our study population, only a minority of patients had previous documented attacks of diverticulitis. Furthermore, a high rate of patients who presented with perforation $(45 \%, 98 / 218)$ presented with this as the initial event of the disease. These findings were similar to those reported in other recent studies [6, 7]. In this series, acute phlegmon was the most frequent presentation of complicated diverticular disease found, as described [8]. Most patients with this mode of presentation can be effectively managed with analgesia, bowel rest and appropriate antibiotics [7], as occurred in our study, in which only patients with persistent signs of sepsis underwent surgery.

Resection and primary anastomosis is the ideal scenario for most patients who present with an attack of complicated diverticulitis because this obviates a second operation such as reversal of Hartmann's. This is usually not possible because of local sepsis but should be the gold 
standard if possible [9]. Treatment of patients with a diverticular abscess depends on magnitude and location of the abscess in addition to the patient's clinical condition at the time of diagnosis [10]. Small pericolic abscesses can usually be resolved with antibiotic therapy and bowel rest. Our study confirmed this fact. For a patient with a large pericolic or distant abscess that does not resolve with medical treatment two options are available, percutaneous or surgical drainage. The potential advantage of percutaneous drainage is stabilization of the patient and the reduction of the abdomino-pelvic inflammation; this should allow a safer resection and clear the area for a primary pelvic anastomosis without diversion [11]. This approach was used in two of our patients that had distant collections. In the present study, a selective operative strategy was used in the treatment of the diverticular abscesses. Thus, among the patients who underwent surgery, a primary anastomosis was selectively performed in the management of well localized collections whereas in the remaining abscesses a Hartmann procedure was performed. Prompt emergency surgery is indicated for patients with diffuse peritonitis, but the optimal surgical approach remains controversial. The procedure of choice in this situation is immediate resection of the septic focus [12]. Following resection, the remaining bowel can be approached in various ways. Some authors $[13,14]$ propose a primary anastomosis wherever possible for patients with all stages of diverticular disease, in the interests of improved outcome, less cost and elimination of a potentially difficult stoma closure after Hartmann's procedure. In agreement with other authors [15-17], we have favored the use of a Hartmann procedure in most patients (96\%) who present with generalized intraperitoneal contamination.

The management of diverticular obstruction can be particularly difficult, but few studies of this complicated problem have been reported. When the obstruction is partial and resolves quickly after hydration and nasogastric decompression, a carefully monitored attempt at mechanical preparation of the bowel is appropriate, but special caution is necessary to avoid inadvertent perforation [16]. Two of our patients with obstruction who were treated conservatively underwent an elective resection at a later, separate hospitalization. The possibility that the obstruction is caused by carcinoma rather than diverticular disease must be considered. The main surgical approach to patients with complete large bowel obstruction caused by diverticular stricture is Hartmann's resection [10]. Nevertheless, in selected patients a primary anastomosis may be performed $[8,16]$. In our experience, this latter surgical approach was performed in two patients. In agreement with several reports $[6,8,18]$, we found that almost all fistulas were amenable to resection and primary anastomosis.

Morbidity is a significant problem after the treatment of complicated diverticulitis, which reflects the seriousness of the disease and the poor general condition of the patients. Our overall morbidity rate of $30 \%$ was similar to that described in other studies $[6,8]$, whereas our major morbidity rate was lower than that reported in a previous study [16]. Considering only patients who underwent surgical treatment, the postoperative morbidity rate obtained in this study was clearly higher to that mentioned in several reports $[7,9,15]$. We also identified a significant correlation between perforated diverticulitis and increased morbidity, as it was confirmed in a previously study [16]. Overall mortality rates in studies on patients with complicated diverticular disease have ranged from 6 to $11 \%[6,8,19]$. In our study, we found a figure that was below of the above mentioned range. Nevertheless, our postoperative mortality rate of $6 \%$ was in agreement with that quoted in earlier studies $[7,9,15,19]$. The diverticular perforation itself and the abdominal infection promoted thereby are very important determinants of the outcome. Thus, in accordance with recent studies $[6,16]$ we observed that the majority of deaths occurred in patients who presented with perforation. Persistent sepsis was the main cause of death, as mentioned $[19,20]$. In older patients with perforated diverticulitis, generalized peritonitis occurs more frequently than localized peritonitis, as a pericolic or pelvic abscess [21]. Regarding this subject in our study, like others $[6,19,22]$, we found that older age together with coexistent medical illness were all significantly associated with a poor outcome. In our experience, a high incidence of postoperative wound infection was found, which was clearly higher to that reported in earlier studies $[4,16,19]$. Given this high incidence and in order to avoid wound dehiscence, a careful wound closure and the use of tension relief sutures in this patient population is recommended.

In conclusion, in our patient population acute diverticulitis was not a good predictor of the development of further complications from diverticular disease as only a minority of patients with these complications had previous documented episodes of diverticulitis. The results of our experience and others' support the fact that each patient hospitalized needs to be managed on a case-by-case basis. Thus, patients managed initially with conservative treatment need to be carefully followed for any sign that indicates this treatment is failing. Among patients re- 
quiring surgery for diverticular disease, those with localized inflammation only, including small abscesses, could be managed with resection and primary anastomosis without diversion. Similarly, most patients with fistulas can be managed on an elective basis with primary anastomosis without diversion. Patients with large or distant abscesses should be managed in a selective fashion. If possible, percutaneous drainage of abscesses should be used in such patients to convert their status from an emergent to an elective one. In patients with abscess requiring operation, a primary anastomosis with or without diversion can be selectively performed in the management of well-localized collections whereas in the remaining abscesses a Hartmann procedure should be performed. Patients with complete large bowel obstruc- tion caused by diverticular stricture can be treated by Hartmann's resection, although in selected patients a primary anastomosis may be performed. Patients with peritonitis are best served with resection, washout and diversion. This study also showed that acute diverticulitis requiring hospitalization continues to carry a persistently high mortality rate for patients with septic complications. This mortality is particularly high in old patients with significant preexisting comorbidities. In these patients, overwhelming sepsis is the major factor contributing to postoperative death. To achieve improvements in outcome, a selective therapeutic approach should be considered, choosing the more adequate surgical procedure for each complication of diverticular disease.

\section{References}

$>1$ Parks TG: Natural history of diverticular disease of the colon. Clin Gastroenterol 1975; 4:53-69.

-2 Aydin HN, Remzi FH: Diverticulitis: when and how to operate. Dig Liver Dis 2004;36: 435-445.

$\checkmark 3$ Rothenberger DA, Wiltz O: Surgery for complicated diverticulitis. Surg Clin N Am 1993 73:975-992.

4 Ferzoco LB, Raptopoulos V, Silen W: Acute diverticulitis. N Engl J Med 1998;338:15211526.

5 Farmakis N, Tudor RG, Keighley MRB: The 5 -year natural history of complicated diverticular disease. Br J Surg 1997;81:733-735.

6 Chapman J, Davies M, Wolf B, Dozois E, Tessier D, Harrington J, Larson D: Complicated diverticulitis: is it time to rethink the rules? Ann Surg 2005;242:576-583.

7 Salem TA, Molloy RG, O’Dwyer PJ: Prospective study on the management of patients with complicated diverticular disease. Colorectal Dis 2005;8:173-176.

8 Tudor RG, Farmakis N, Keighley MRB: National audit of complicated diverticular disease: analysis of index cases. Br J Surg 1994; 81:730-732.

$\checkmark 9$ Bahadursingh AM, Virgo KS, Kaminski DL, Longo WE: Spectrum of disease and outcome of complicated diverticular disease. Am J Surg 2003;186:696-701.
10 Wong WD, Wexner SD, Lowry A, Vernava A III, Burnstein M, Denstman F, Fazio V, Kerner B, Moore R, Oliver G, Peters W, Ross T, Senatore P, Simmang C: The standards task force of the American society of colon and rectal surgeons: practice parameters for the treatment of sigmoid diverticulitis - supporting documentation. Dis Colon Rectum 2000;43:289-297.

11 Kaiser AM, Jiang JK, Lake JP, Ault G, Artinyan A, González-Ruiz C, Essani R, Beart RW Jr: The management of complicated diverticulitis and the role of computed tomography. Am J Gastroenterol 2005;100:910-917.

12 Krukowski ZH, Matheson NA: Emergency surgery for diverticular disease complicated by generalized and faecal peritonitis: a review. Br J Surg 1984;71:921-927.

13 Wedell J, Banzhaf G, Chaoui R, Fischer R, Reichmann J: Surgical management of complicated colonic diverticulitis. Br J Surg 1997; 84:380-383.

14 Richter S, Lindemann W, Kollmar O, Pistorius GA, Maurer CA, Schilling MK: Onestage sigmoid colon resection for perforated sigmoid diverticulitis (Hinchey stages III and IV). World J Surg 2006;30:1027-1032.

15 Belmonte C, Klas JV, Perez JJ, Wong D, Rothenberger DA, Goldberg SM, Madoff RD: The Hartmann procedure: first choice or last resort in diverticular disease? Arch Surg 1996;131:612-617.
16 Schwesinger WH, Page CP, Gaskill HV III, Steward RM, Chopra S, Strodel WE, Sirinek KR: Operative management of diverticular emergencies: strategies and outcomes. Arch Surg 2000;135:558-563.

17 Mäkelä J, Vuolio S, Kiviniemi H, Laitinen S: Natural history of diverticular disease. When to operate? Dis Colon Rectum 1998; 41:1523-1528.

18 Carvajal Balaguera J, Camunas Segovia J, Pena Gamarra L, Oliart Delgado de Torres S, Martin Garcia-Almenta M, Viso Ciudad S, Fernández IP, Gomez Maestro P, Cerquella Hernández C: Colovesical fistula complicating diverticular disease: one-stage resection. Int Surg 2006;91:17-23.

19 Elliot TB, Yego S, Irvin TT: Five-year audit of the acute complications of diverticular disease. Br J Surg 1997;84:535-539.

20 Mäkelä J, Kiviniemi H, Laitinen S: Prevalence of perforated sigmoid diverticulitis is increasing. Dis Colon Rectum 2002;45:955961.

21 Watters JM, Blaslee JM, March RJ, Redmond ML: The influence of age on the severity of peritonitis. Can J Surg 1996;39:142-146.

22 Pisanu A, Cois A, Uccheddu A: Surgical treatment of perforated diverticular disease: evaluation of factors predicting prognosis in the elderly. Int Surg 2004;89:35-38. 